\title{
Higher Procalcitonin Level in Cerebrospinal Fluid than in Serum Is a Feasible Indicator for Diagnosis of Intracranial Infection
}

\author{
Huajun Wang
}

\begin{abstract}
Objective: To explore the value of the difference in procalcitonin (PCT) levels in serum and cerebrospinal fluid (CSF) for diagnosing intracranial infection in post-neurosurgical patients.

Methods: Patients who were treated at our hospital after craniotomy from January 2015 to January 2019 were enrolled in this study. Twenty patients eventually diagnosed with intracranial infection were included in a study group and 22 patients with no intracranial infection were included in a control group. A t-test was used to compare the differences between serum and CSF PCT levels of PCT, and the diagnostic value of PCT was evaluated by receiver operating characteristic (ROC) curve analysis.

Results: The serum PCT levels in the study and control groups were $0.10 \pm 0.03 \mathrm{ng} / \mathrm{mL}$ and $0.09 \pm 0.03 \mathrm{ng}$ / $\mathrm{mL}$, respectively, and they were not substantially different between the groups. The CSF PCT level in the study group was substantially higher than that in the control group, with values of $0.13 \pm 0.03 \mathrm{ng} / \mathrm{mL}$ and $0.07 \pm 0$. $02 \mathrm{ng} / \mathrm{mL}$, respectively. The CSF/serum PCT ratio in the study group was substantially higher than that in the control group, with values of $1.31 \pm 0.19$ and $0.79 \pm 0.23$, respectively. The areas under the ROC curve for serum PCT, CSF PCT and the CSF/serum PCT ratio were 0.56,0.92, and 0.95 , respectively, resulting in a substantial difference among the three groups.

Conclusion: CSF PCT may be a valuable marker for diagnosing intracranial infection in patients after neurosurgery; in particular, the specificity of CSF PCT is higher if the CSF PCT level is higher than the serum PCT level.
\end{abstract}

Keywords: cerebrospinal fluid; intracranial infection; procalcitonin; serum

$\mathbf{S}$ ECONDARY INTRACRANIAL INFECTION after craniotomy is common complication, with an incidence ranging from $1 \%$ to $10 \%[1,2]$, and it is an important reason for increases in hospital costs and hospitalization time. The diagnostic criteria for intracranial infection include elevated body temperature, clinical symptoms, and laboratory examination findings, but these findings lack specificity. In addition, it is difficult to distinguish post-neurosurgical bacterial meningitis (PNBM) from aseptic meningitis, which is caused by an aseptic inflammatory response to the products of sloughing tissue, tumor antigen hemolysis, bone dust, and hemolysis. If the diagnosis is delayed, intracranial infection is likely to cause complications in the central nervous system and seriously affect the prognosis. Therefore, rapidly distinguishable and accurate biomarkers can improve the prognosis of patients with PNBM. Recent studies have found that procalcitonin (PCT) is an indicator of systemic bacterial infection that can be used to diagnose intracranial infections [3-6]. However, other scholars believe that cerebrospinal fluid (CSF) PCT is derived from the serum as a result of the destruction of the blood-brain barrier; it is affected by surgical trauma, anesthesia, and other factors and it cannot be used to differentiate infectious fever from noninfectious fever [7]. In summary, the usefulness of CSF PCT in the diagnosis of intracranial infection has not been demonstrated fully. Whether PCT is locally produced in the central nervous system is a matter of debate. The purpose of this study was to compare serum and CSF PCT levels to investigate the value of PCT for diagnosing intracranial infection in patients with PNBM.

\section{Patients and Methods}

Patients

This prospective observational cohort study was conducted from January 2015 to January 2019 in the intensive

Department of Intensive Care Unit, Yinzhou People's Hospital, Ningbo University Medical College, Ningbo, Zhejiang, China. 
care unit (ICU) of Ningbo Yinzhou People's Hospital. All patients ( $>18$ years) who had undergone surgery 48-72 hours previously were recruited to participate in this study. The study group was diagnosed with intracranial infection, and the control group included patients in whom intracranial infections had been excluded. Twelve males and eight females were included in the study group, with an age range from 38 to 83 years (average: 67.20 12.22 years). Twelve males and 10 females were included in the control group, with an age range from 42 to 86 years (average: $66.89 \pm 11.83$ years). All patients had cerebral hemorrhage, including 29 cases of hypertensionassociated hemorrhage, six cases of a ruptured aneurysm, and seven cases of a ruptured vascular malformation.

\section{Methods}

For patients who were suspected of having an intracranial infection after neurosurgery, informed consent was obtained, a lumbar puncture was performed, and a CSF sample was taken to the laboratory for routine CSF analysis, biochemical analysis, culturing, and the measurement of CSF PCT levels. Serum PCT levels were measured simultaneously. All procedures were based on the assumption of an absence of bacterial infection.

\section{Diagnostic criteria for intracranial infection}

The diagnostic standards in this study included the following: the presence of clinical symptoms of intracranial infection; a positive CSF culture; a CSF white blood cell (WBC) count $\geq 1,000$ cells per microliter and a polykaryocyte percentage $\geq 75 \%$; and a CSF glucose level $<2.5 \mathrm{mmol} / \mathrm{L}$ or a CSF/serum glucose ratio $<0.4$ [8]. The diagnosis of intracranial infection was made individually in patients meeting the second criterion. Intracranial infection was also diagnosed in patients with negative CSF culture results but who met the other three diagnostic criteria. Those who had one or more of the criteria but did not meet the second criterion or all three other diagnostic criteria were considered as the control group. Patients with none of the inclusion criteria were excluded from our study.

\section{Detection methods}

All patients who underwent craniotomy also underwent a lumbar puncture for the collection of CSF specimens. Fifteen milliliters of CSF were collected in a sterile test tube, $5 \mathrm{~mL}$ of which was centrifuged for five minutes $(3,000 \mathrm{r} / \mathrm{rain})$, after which PCT was detected. The residual CSF samples were immediately sent to the clinical laboratory, and a VITROS 5. 1 FS automatic chemistry analyzer (Diamond Diagnostics, Citrus Heights, CA) was used to detect the CSF components (protein, chloride, and glucose levels). Procalcitonin was detected using an enzyme-linked fluorescence method (bioMérieux Marcy L'Etoile, France); the lower limit of positivity was $0.05 \mathrm{ng} / \mathrm{mL}$, as determined by the manufacturer. Blood samples were obtained by peripheral venipuncture under sterile conditions, and the time interval between CSF and blood sample collection in the same patient was $<30$ minutes.

\section{Statistical analysis}

The statistical analysis was performed using SPSS 20.0 software (IBM Corp., Armonk, NY). Normally distributed data and count data were compared by t-tests and $\chi^{2}$ tests, respectively. Receiver operating characteristic (ROC) curve analysis was used to analyze the diagnostic efficacy of PCT. A value of $\mathrm{P}<0.05$ was considered statistically significant.

\section{Results}

Comparison of general data between the two groups of patients

No differences were observed in age, body temperature, gender, APACHE II score, serum C-reactive protein (CRP) levels or CSF protein levels, leukocyte counts, and chloride levels between the two groups, but an important difference was found in CSF glucose levels between the two groups $(\mathrm{p}>0.05$, Table 1).

\section{Distribution of cerebrospinal fluid pathogens in the study group}

Pathogenic bacteria were cultured from the cerebrospinal fluid of seven patients in the study group, with a positivity rate of $35 \%$. A total of nine strains of pathogenic bacteria were detected, including Staphylococcus aureus, Enterococcus faecium, epidermis Staphylococcus aureus, Acinetobacter baumannii, Klebsiella pneumoniae, Pseudomonas aeruginosa, and Escherichia coli, among which gram-positive bacteria accounted for $44 \%$, and gram-negative bacteria accounted for $56 \%$. Samples from two of the patients were positive for the presence of two strains of pathogenic bacteria (Table 2).

\section{Measurement of PCT levels}

The PCT positivity rates in the CSF in the study and control groups were $100 \%$ and $73 \%$, respectively, when the positive

Table 1. General Data from the Two Groups at Admission

\begin{tabular}{|c|c|c|c|}
\hline & Study group & Control group & $p$ \\
\hline Gender (male/female) & $12 / 8$ & $12 / 10$ & 0.83 \\
\hline Age (y) & $67.20 \pm$ & $66.89 \pm$ & 0.95 \\
\hline APACHE II score & $21.47 \pm$ & $20.89 \pm$ & 0.66 \\
\hline Serum CRP (mg/L) & $30.88 \pm$ & $60.62 \pm$ & 0.08 \\
\hline CSF glucose $(\mathrm{mmol} / \mathrm{L})$ & $3.00 \pm$ & $4.74 \pm$ & 0.01 \\
\hline CSF protein $(\mathrm{mg} / \mathrm{L})$ & $1,293.07 \pm 1,123.22$ & $1,323.89 \pm 1,688.29$ & 0.96 \\
\hline CSF leukocyte count $\left(10^{*} 6 / \mathrm{L}\right)$ & $227.13 \pm 319.21$ & $169.00 \pm 326.67$ & 0.68 \\
\hline CSF chloride $(\mathrm{mmol} / \mathrm{L})$ & $128.00 \pm \quad 9.58$ & $134.11 \pm \quad 6.96$ & 0.08 \\
\hline Temperature $\left({ }^{\circ} \mathrm{C}\right)$ & $38.78 \pm$ & $38.26 \pm$ & 0.06 \\
\hline
\end{tabular}

$\mathrm{CRP}=\mathrm{C}$ reactive protein; $\mathrm{CSF}=$ cerebrospinal fluid. 
Table 2. Structure of Pathogens Isolated FROM PATIENTS WITH INTRACRANIAL INFECTION

\begin{tabular}{lcc}
\hline Causative agent & $\begin{array}{c}\text { Absolute } \\
\text { number } \\
\text { of pathogens }\end{array}$ & $\begin{array}{c}\text { Composition } \\
\text { ratio (\%) }\end{array}$ \\
\hline Gram-negative bacteria & 5 & 56 \\
$\quad$ Acinetobacter baumannii & 2 & 22.2 \\
Klebsiella pneumoniae & 1 & 11.1 \\
Pseudomonas aeruginosa & 1 & 11.1 \\
Escherichia coli & 1 & 11.1 \\
Gram-positive bacteria & 4 & 44 \\
$\quad$ Staphylococcus aureus & 1 & 11.1 \\
Staphylococcus epidermidis & 2 & 22.2 \\
Enterococcus faecium & 1 & 11.1 \\
Fungi & 0 & 0 \\
Tubercle bacillus & 0 & 0 \\
Total & 9 & 100 \\
\hline
\end{tabular}

reference value was $0.05 \mathrm{ng} / \mathrm{mL}$ or higher, which resulted in a statistically significant difference between the two groups $(\mathrm{p}<0.05$, Table 3$)$. A statistically significant difference in CSF PCT levels was observed between the two groups $(\mathrm{p}<0.05$, Table 4). No significant difference in serum PCT levels was found between the two groups ( $p>0.05$, Table 4$)$.

\section{Analysis of the efficacy of PCT in the diagnosis of intracranial infection by ROC curve}

The areas under the ROC curves of serum PCT, CSF PCT, and the CSF/serum PCT ratio were 0.56, 0.92 and 0.95 , respectively (Fig. 1), resulting in a substantial difference among these factors (Table 5). When the cutoff value of PCT for a diagnosis of intracranial infection was $>0.05 \mathrm{ng} / \mathrm{mL}$, the diagnostic sensitivity of CSF PCT was $100 \%$ and the specificity was $<27 \%$; furthermore, the diagnostic sensitivity of serum PCT was $90 \%$, and the specificity was $5 \%$. When the cutoff value was increased to $0.09 \mathrm{ng} / \mathrm{mL}$, the sensitivity of CSF PCT for diagnosing intracranial infection was $80 \%$ and the specificity increased to $82 \%$. The specificity and sensitivity of serum PCT were $27 \%$ and $60 \%$, respectively. When a CSF/serum PCT ratio $>1$ was used to diagnose intracranial infection, the specificity and sensitivity were $86 \%$ and $95 \%$, respectively.

\section{Difference in the CSF/serum PCT ratio between the two groups}

The CSF/serum PCT ratio was $(1.31 \pm 0.19)$ in the study group and $(0.79 \pm 0.23)$ in the control group, resulting in a

Table 3. Positive Rate of CSF for PCT IN THE TwO GROUPS

\begin{tabular}{lcccc}
\hline & \multicolumn{2}{c}{ CSF $P C T$} & & Positive rate \\
\cline { 2 - 3 } Group & $\geq 0.05 n g / m L$ & $<0.05 n g / m L$ & & $\%$ \\
\hline Study group & 20 & 0 & & 100 \\
Control group & 16 & 6 & & 73 \\
p value & & & & 0.02 \\
\hline
\end{tabular}

$\mathrm{CSF}=$ cerebrospinal fluid $\mathrm{PCT}=$ procalcitonin.
Table 4. Serum and CSF PCT Data of the Two Groups

\begin{tabular}{lcc}
\hline Group & CSF PCT $(\mathrm{ng} / \mathrm{mL})$ & Serum PCT $(\mathrm{ng} / \mathrm{mL})$ \\
\hline Study group & $0.13 \pm 0.03$ & $0.10 \pm 0.03$ \\
Control group & $0.07 \pm 0.02$ & $0.09 \pm 0.02$ \\
p value & 0.01 & 0.86 \\
\hline
\end{tabular}

$\mathrm{CSF}=$ cerebrospinal fluid; $\mathrm{PCT}=$ procalcitonin.

statistically significant difference between the two groups $(\mathrm{p}=0.01$, Table 6).

\section{Discussion}

Intracranial infection is a common complication of postoperative cerebral hemorrhage, but the diagnosis of intracranial infection lacks specific indicators or examinations and mainly relies on CSF examinations [9]. The sensitivity of routine and biochemical CSF examinations is high, but the specificity is low, which is consistent with the results of this study. The bacterial culture of CSF remains the gold standard for the diagnosis of intracranial infection, but the positivity rate in our study was only $35 \%$, and the culturing requires a long time, which is not conducive to the early diagnosis of intracranial infection. Therefore, it is important to identify sensitive, specific, simple, and easily detectable indicators for the clinical diagnosis and treatment of intracranial infections. Procalcitonin is a prepeptide of calcitonin. When the body becomes infected with bacteria, the concentration of PCT in the blood increases within a short time and the PCT concentration is positively correlated with the severity of the infection. Therefore, PCT is a key indicator for the diagnosis of bacterial infection and has been used widely in clinical practice $[10,11]$. Recent studies have also shown that elevated PCT levels in the serum and CSF may be used for the diagnosis of intracranial infection. Other studies have shown that CSF PCT has better sensitivity and specificity than serum PCT [12-15]. Additionally, studies have suggested that increased CSF PCT is a non-specific finding, even though the brain tissue itself does not produce synthetic secretions $[16,17]$. However, the role of CSF PCT in the diagnosis of intracranial infection and whether the brain tissue itself secretes PCT remain unclear.

This study found that the CSF PCT levels in the infected group were substantially higher than those in the nonintracranial infection group. However, no difference was observed in serum PCT levels between the two groups. Furthermore, we found that the area under the ROC curve for CSF PCT was 0.92, which was substantially different from the area under the ROC curve for serum PCT (by 0.56). These findings suggest that increases in CSF PCT levels can be used for the diagnosis of intracranial infection, which is similar to the conclusions of many other studies $[13,14,18]$. However, in this study, serum PCT did not show good diagnostic value for intracranial infection, which is consistent with the results of Choi et al.'s study [19]. We hypothesized that because intracranial infection is a localized rather than a systemic infection, the difference in serum PCT levels would not be significant. Although many studies (including a metaanalysis) have suggested that CSF PCT is a good potential indicator for the diagnosis of intracranial infection, reference 


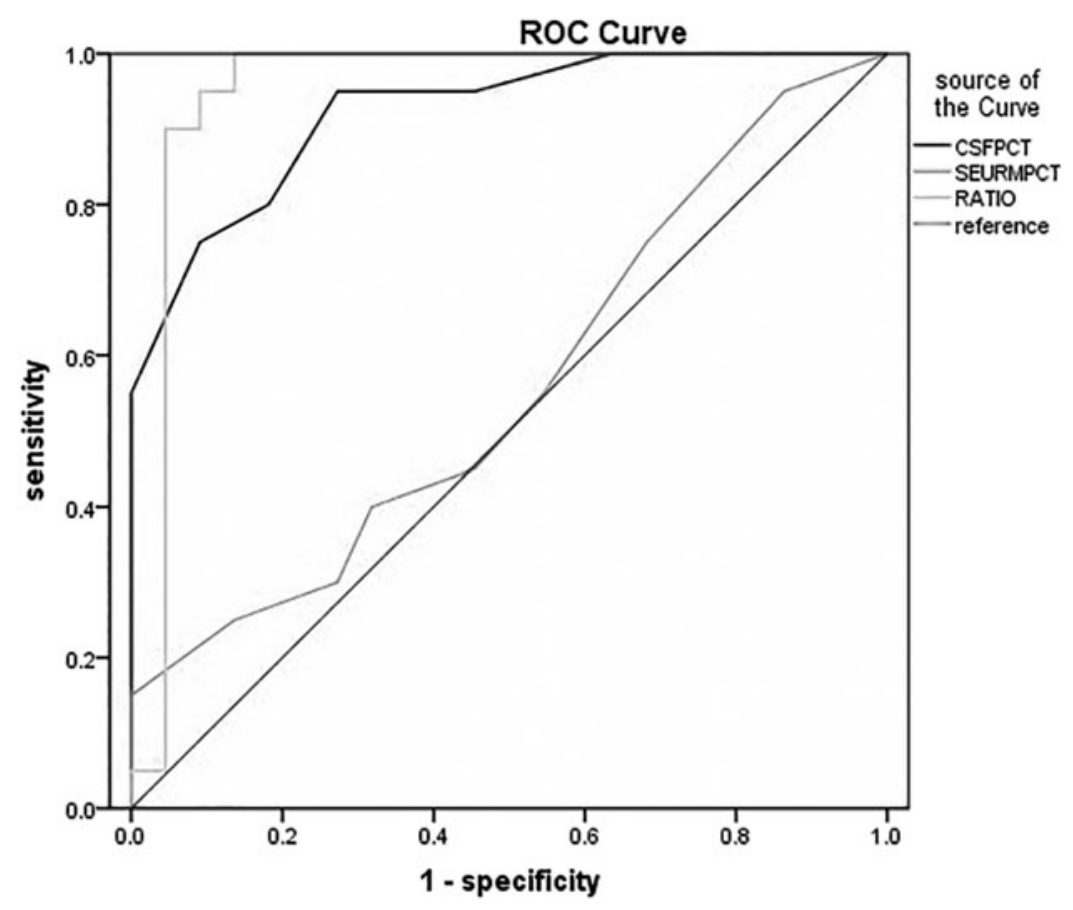

FIG. 1. Receiver operating characteristic (ROC) analysis illustrating that the cerebrospinal fluid (CSF) procalcitonin (PCT) and CSF/serum PCT ratio are significant predictors of the post-neurosurgical bacterial meningitis (PNBM) (area under the curve $[\mathrm{AUC}]=0.92, \mathrm{p}<0.005$, and $\mathrm{AUC}=0.95, \mathrm{p}<0.005$, respectively).

values have not been determined [20]. At present, most studies use the reference value for serum PCT $(0.05 \mathrm{ng} / \mathrm{mL})$. However, this study found that the positivity rate of CSF PCT in the control group was $73 \%$, the sensitivity of CSF PCT in the diagnosis of intracranial infection was $100 \%$, and the specificity was less than $27 \%$; obviously, these results do not meet clinical requirements. The Guidelines for HealthcareAssociated Ventriculitis and Meningitis have advised that increased CSF PCT levels may indicate intracranial infection [21]. However, the optimal cutoff value for PCT using the Youden index was $0.09 \mathrm{ng} / \mathrm{mL}$, which yielded a sensitivity for diagnosing intracranial infection of $80 \%$ and a specificity of $82 \%$, which meet clinical needs. This threshold is similar to those in several other studies [22-24].

When CSF and serum PCT levels were compared simultaneously, we found that most patients with intracranial infections had higher CSF PCT levels than serum PCT levels, and the CSF/serum PCT ratio was different between the two groups. When intracranial infection was diagnosed using a $\mathrm{CSF} /$ serum PCT ratio $>1$, the area under the ROC curve was $95 \%$, the specificity was $95 \%$, and the sensitivity was $86 \%$. These sensitivity and specificity values meet clinical needs.

TABle 5. Area under the ROC Curve

\begin{tabular}{lcc}
\hline Group & Area under ROC curve & $p$ \\
\hline Serum PCT & 0.56 & \\
CSF PCT & 0.92 & $0.01 *$ \\
CSF/serum PCT ratio & 0.95 & $0.02 *$ \\
\hline
\end{tabular}

*Indicates a comparison with serum PCT.

$\mathrm{ROC}=$ receiver operating characteristic; $\mathrm{CSF}=$ cerebrospinal fluid; $\mathrm{PCT}=$ procalcitonin
To the best of our knowledge, this is the first study to diagnose intracranial infection in patients with PNBM using the $\mathrm{CSF} /$ serum PCT ratio. Clearly, the nerve tissue itself also stimulates the secretion of PCT because of inflammation, and the CSF PCT is not derived from serum PCT. Therefore, the presence of higher CSF PCT levels than serum PCT levels can be used for the diagnosis and differential diagnosis of intracranial infection, and the specificity of this method is better than that of the use of the CSF PCT level alone.

In conclusion, we suggest that CSF PCT is highly sensitive for the diagnosis of intracranial infections, but it lacks specificity, which is consistent with the Guidelines for HealthcareAssociated Intracranial Infections developed by the American Society of Infectious Diseases in 2017. However, the specificity of CSF PCT increases with increasing cutoff values or when CSF PCT levels are higher than serum PCT levels.

This is an exploratory study and has several limitations. First, the clinical status of patients in the intensive care unit is complex, and they can have various infections. The sample size of the enrolled group was small, and the positivity rate of CSF culture was too low. We did not have enough samples to compare the differences in CSF PCT among different pathogen types. We will try to collect more samples from multiple

Table 6. CSF/Serum PCT Ratio of Two Groups

\begin{tabular}{lc}
\hline Group & CSF/Serum $P C T$ ratio \\
\hline Study group & $1.31 \pm 0.19$ \\
Control group & $0.79 \pm 0.23$ \\
p value & 0.01 \\
\hline
\end{tabular}

$\mathrm{CSF}=$ cerebrospinal fluid; $\mathrm{PCT}=$ procalcitonin. 
centers. At the same time, the detection rate of pathogenicity can be increased by the second generation sequencing method of the CSF; we will dynamically observe the changes in serum PCT and CSF PCT before and after anti-infection therapy and explore the guiding effect of CSF on antiinfective efficacy. We plan to explore whether the increase in PCT in CSF is related to bacterial endotoxin, tumor necrosis factor, interleukin-1, interleukin-6, and other inflammatory factors. Further research is needed to verify the usefulness of measuring PCT for the early diagnosis of intracranial infections in patients undergoing craniotomy.

\section{Acknowledgments}

I thank all of the patients, doctors, and staff involved in the study. I also thank my family for their support.

\section{Funding Information}

This work did not require any funding.

\section{Author Disclosure Statement}

No competing financial interests exist.

\section{References}

1. Shi ZH, Xu M, Wang YZ, et al. Post-craniotomy intracranial infection in patients with brain tumors: A retrospective analysis of 5723 consecutive patients. Br J Neurosurg 2017; 31:5-9.

2. Srinivas D, Veena Kumari HB, et al. The incidence of postoperative meningitis in neurosurgery: An institutional experience. Neurology India 2011;59:195-198.

3. Gautam-Goyal P, Malhotra P, Epstein M, et al. Can procalcitonin be used as a reliable marker for infectious fever in patients with intracranial hemorrhage?JWorld Neurosurg 2018;116:e968-e974.

4. Tomio R, Akiyama T, Shibao S, Yoshida K. Procalcitonin as an early diagnostic marker for ventriculoperitoneal shunt infections. Surg Infect 2013;14:433-436.

5. Konstantinidis T, Cassimos D, Gioka T, et al. Can Procalcitonin in cerebrospinal fluid be a diagnostic tool for meningitis? Clin Lab Anal 2015;29:169-174.

6. Zhang G, Yang C, Kang X, et al. The combination of cerebrospinal fluid procalcitonin, lactate, interleukin- 8 and interleukin-10 concentrations for the diagnosis of postneurosurgical bacterial meningitis: A prospective study. Ann Clin Biochem 2019;56:133-140.

7. Halvorson K, Shah S, Fehnel C. Procalcitonin is a Poor Predictor of Non-Infectious Fever in the Neurocritical Care Unit. Neurocrit Care 2017;27:237-241.

8. Gray LD, Fedorko DP. Laboratory diagnosis of bacterial meningitis. Clin Microbiol Rev 1992;5:130-145.

9. White K, Ostrowski K, Maloney S, Norton R. The utility of cerebrospinal fluid parameters in the early microbiological assessment of meningitis. Diagn Microbiol Infect Dis 2012; 73:27-30.

10. Angeletti S, Spoto S, Fogolari M, et al. Diagnostic and prognostic role of procalcitonin (PCT) and MR-pro-adrenomedullin (MR-proADM) in bacterial infections. APMIS 2015;123:740 748.

11. Miedema KG, de Bont ES, Elferink RF, et al. The diagnostic value of CRP, IL-8, PCT, and sTREM-1 in the de- tection of bacterial infections in pediatric oncology patients with febrile neutropenia. Support Care Cancer 2011;19: $1593-1600$.

12. Intan S, Samuel DV. Clinical utility and measurement of procalcitonin. Clin Biochem 2017;38:59-68.

13. Zhu L, Dong L, Li Y, et al. The diagnostic and antibiotic reference values of procalcitonin for intracranial infection after craniotomy. World Neurosurg 2018;11:S1878-8750.

14. Shen HY, Gao W, Cheng JJ, et al. Direct comparison of the diagnostic accuracy between blood and cerebrospinal fluid procalcitonin levels in patients with meningitis. Clin Biochem 2015;48:1079-1082.

15. Zhang L, Ma L, Zhou X , et al. Diagnostic value of procalcitonin for bacterial meningitis in children: a comparison analysis between serum and cerebrospinal fluid procalcitonin levels. Clin Pediatr (Phila) 2019;58:159-165.

16. Shimetani N, Shimetani K, Mori M. Levels of three inflammation markers, C-reactive protein, serum amyloid A protein and procalcitoninin the serum and cerebrospinal fluid of patients with meningitis. Scand J Clin Lab Invest 2001;61:567-574.

17. Jereb M, Muzlovic S, Hojker F, Strle F. Predictive value of serum and cerebrospinal fluid procalcitonin levels for the diagnosis of bacterial meningitis. Infection 2001;29:209212.

18. Yu Y, Li HJ. Diagnostic and prognostic value of procalcitonin for early intracranial infection after craniotomy. Braz J Med Biol 2017;50:e6021.

19. Choi SH, Choi SH. Predictive performance of serum procalcitonin for the diagnosis of bacterial meningitis afterneurosurgery. Infect Chemother 2013;45:308-314.

20. Wei TT, Hu ZD, Qin BD, et al. Diagnostic accuracy of procalcitonin in bacterial meningitis versus nonbacterial meningitis: A systematic review and meta-analysis. Medicine 2016;95:e3079.

21. Tunkel AR, Hasbun R, Bhimraj A, et al. 2017 Infectious Diseases Society of America's Clinical Practice Guidelines for Healthcare Associated Ventriculitis and Meningitis. Clin Infect Dis 2017;64:e34-e65.

22. Chen YM, Xu G, Chen M, Dong BH. Predictive value of procalcitonin level of serum and cerebrospinal fluid for intracranial infections after craniotomy. Chin J Nosocomiol 2015;25:5612-5614.

23. Li Y, Zhang G, Ma R, et al. The diagnostic value of cerebrospinal fluids procalcitonin and lactate for the differential diagnosis of post-neurosurgical bacterial meningitis and aseptic meningitis. Clin Biochem 2015;48:50-54.

24. Alons IM, Verheul RJ, Kuipers I, et al. Procalcitonin in cerebrospinal fluid in meningitis: A prospective diagnostic study. Brain Behav 2016;6:e0545

Address correspondence to: Dr. Huajun Wang Department of Intensive Care Unit Yinzhou People's Hospital Ningbo University Medical College Baizhang East Road 251 Ningbo, Zhejiang China

E-mail:whj269696@163.com 